\title{
Discretization of Compact Riemannian Manifolds Applied to the Spectrum of Laplacian
}

\author{
TATIANA MANTUANO \\ Université de Neuchâtel, Institut de Mathématiques, rue Emile-Argand 13, 2007 Neuchâtel, \\ Switzerland.e-mail: tatiana.mantuano@unine.ch
}

(Received: 29 January 2004; accepted: 6 October 2004)

Communicated by R. Kellerhals (Fribourg)

\begin{abstract}
For $\kappa \geqslant 0$ and $r_{0}>0$ let $\mathcal{M}\left(n, \kappa, r_{0}\right)$ be the set of all connected, compact $n$-dimensional Riemannian manifolds $\left(M^{n}, g\right)$ with Ricci $(M, g) \geqslant-(n-1) \kappa g$ and $\operatorname{Inj}(M) \geqslant r_{0}$. We study the relation between the $k$ th eigenvalue $\lambda_{k}(M)$ of the Laplacian associated to $\left(M^{n}, g\right), \Delta=-\operatorname{div}(\operatorname{grad})$, and the $k$ th eigenvalue $\lambda_{k}(X)$ of a combinatorial Laplacian associated to a discretization $X$ of $M$. We show that there exist constants $c, C>0$ (depending only on $n, \kappa$ and $r_{0}$ ) such that for all $M \in \mathcal{M}\left(n, \kappa, r_{0}\right)$ and $X$ a discretization of $M, c \leqslant \frac{\lambda_{k}(M)}{\lambda_{k}(X)} \leqslant C$ for all $k<|X|$. Then, we obtain the same kind of result for two compact manifolds $M$ and $N \in \mathcal{M}\left(n, \kappa, r_{0}\right)$ such that the Gromov-Hausdorff distance between $M$ and $N$ is smaller than some $\eta>0$. We show that there exist constants $c, C>0$ depending on $\eta, n, \kappa$ and $r_{0}$ such that $c \leqslant \frac{\lambda_{k}(M)}{\lambda_{k}(N)} \leqslant C$ for all $k \in \mathbb{N}$.
\end{abstract}

Mathematics Subject Classification (2000): 58J50, 53C20.

Key words: Laplacian, eigenvalues, discretization, Hausdorff-Gromov distance.

\section{Introduction}

Since the work of Buser in [6], it is known that in order to understand the spectrum of the Laplacian associated to a compact Riemannian manifold, $\Delta=-\operatorname{div}(\mathrm{grad})$, it may be very powerful to discretize the manifold. Using this technique, Buser considered manifolds with Ricci curvature and injectivity radius bounded below and gave an uniform estimate of the spectrum, depending only on these bounds (see Theorem 6.2 in [6]). The estimate of the $k$ th eigenvalue turned out to be very precise for large $k$ 's (i.e. for $k$ larger than a constant proportional to the volume of the manifold). However, for the beginning of the spectrum, the result is not strong enough to decide whether the eigenvalues may be close to zero or not.

Since, this question has been investigated by Brooks [2, 3], Burger [4], and Buser himself in [7]. In these papers, the manifolds were especially, closely related to Cayley graphs of groups or to Schreier graphs associated to a family of covering spaces (in another context see also the work of Kanai [13, 14]).

\footnotetext{
${ }^{\star}$ Supported by Swiss National Science Foundation, grant No. 20-101 469.
} 
The point of view that will interest us here is the one taken up by Chavel in his book [8], where the question of discretization is very well explained and where he studies in particular the case of isoperimetric inequalities ([8], Chapter V) and Sobolev inequalities ([8], Chapter VI). This book will be the main reference for this paper.

The purpose of this note is to compare the spectrum of a compact Riemannian manifold $\left(M^{n}, g\right)$ to the spectrum of the combinatorial Laplacian of an associated discretization $X$ (defined as in [8]). More precisely, if $\mathcal{M}\left(n, \kappa, r_{0}\right)$ denotes the set of all compact $n$-dimensional Riemannian manifolds with Ricci curvature and injectivity radius uniformly bounded below (i.e. with Ricci $(M, g) \geqslant-(n-1) \kappa g$, $\kappa \geqslant 0$ and $\operatorname{Inj}(M) \geqslant r_{0}>0$, see Definition 3.6), we will show in Theorem 3.7 that there exist positive constants $c, C$ (depending only on $n, \kappa, r_{0}$ ) such that for all manifolds $M$ in $\mathcal{M}\left(n, \kappa, r_{0}\right)$ and $X$ a discretization associated to $M$, we have

$$
c \leqslant \frac{\lambda_{k}(M)}{\lambda_{k}(X)} \leqslant C
$$

for all $k<|X|$. Remark that $|X|$ behaves as the volume of $M$.

After defining precisely in Section 2 the notion of discretization and the Laplacian related to it, Section 3 will be concerned with the proof of this result.

In Section 4, as a corollary of our result, we present a very simple proof of Theorem 1 of Brooks in [2], which says that the first nonzero eigenvalue of a tower of covering spaces of a compact manifold goes to zero if and only if the Cheeger constant of the discretizations associated to the covering spaces does. In fact, we prove that the $k$ th eigenvalue of a tower of covering spaces of a compact manifold goes to zero if and only if the $k$ th eigenvalue of the discretizations associated to the covering spaces does, which implies obviously the result of Brooks (see Theorem 4.1). Note that our proof avoids integral geometry and some not obvious considerations on the boundary of Dirichlet's fundamental domains.

In Section 5, we compare the spectrum of two compact Riemannian manifolds $M \in \mathcal{M}\left(m, \kappa, r_{0}\right)$ and $N \in \mathcal{M}\left(n, \kappa, r_{0}\right)$ which are close with respect to the Gromov-Hausdorff distance (see Theorem 5.1). In particular, as $m=n$ we show in Corollary 5.2 that we have an uniform control $c \leqslant \frac{\lambda_{k}(M)}{\lambda_{k}(N)} \leqslant C$ for all $k$ and where $c$, $C>0$ depend on $n, \kappa, r_{0}$ and on the Gromov-Hausdorff distance between $M$ and $N$ (for the behaviour of the spectrum under convergence of manifolds with respect to the Gromov-Hausdorff distance see Section 7 of [10]).

We conclude this note with Section 6, where we give an example to show that the assumption on the injectivity radius is essential in Theorem 5.1; the spectra of two manifolds with Ricci curvature bounded below and arbitrarily Gromov-Hausdorff close may strongly differ.

\section{Spectrum of Roughly Isometric Graphs}

Let $X=(V, E)$ be a finite graph, where $V$ denotes the set of vertices and $E$ the set of edges, and consider the path metric on this graph so that it becomes a metric 
space (see [8], p. 140). Denote by $N(v)$ the set of neighbours of $v \in V$, that is to say the set of vertices at distance 1 from $v$ for the path metric. We will refer to $m(v)$ as the number of neighbours of $v \in V$ and to $v_{X}$ as an upper bound for $m(v)$ (i.e. $v_{X}$ is such that for all $\left.v \in V, m(v) \leqslant v_{X}\right)$.

For such a finite graph, we can define a combinatorial Laplacian as in [15] (Section 4.2) and the spectrum of this Laplacian will be denoted by

$$
\operatorname{Spec}(X)=\left\{0=\lambda_{0}(X) \leqslant \lambda_{1}(X) \leqslant \cdots \leqslant \lambda_{l}(X)\right\}
$$

where $l=|X|-1$ and $|X|$ denotes the number of vertices of the graph. We have the following variational characterization of $\operatorname{Spec}(X)$ (see [1], p. 268). For a function $f: V \rightarrow \mathbb{R}$, consider the Rayleigh quotient of $f$,

$$
R(f)=\frac{\|\mathrm{d} f\|^{2}}{\|f\|^{2}}
$$

where $\|\mathrm{d} f\|^{2}=\sum_{v \in V}|\mathrm{~d} f|^{2}(v)=\sum_{v \in V} \sum_{w \in N(v)}|f(w)-f(v)|^{2}$ and $\|f\|^{2}=$ $\sum_{v \in V} f^{2}(v)$. Then, for any $(k+1)$-dimensional vector subspace $W^{(k+1)}$ of the vector space $\mathcal{F}(V)=\{f: V \rightarrow \mathbb{R}\}$, the $k$ th eigenvalue of $X$ satisfies

$$
\lambda_{k}(X) \leqslant \sup \left\{R(f): f \in W^{(k+1)}, f \neq 0\right\}
$$

So, if $\Lambda(W)$ denotes the supremum of Rayleigh quotients of nonzero functions in $W$ (i.e. $\Lambda(W)=\sup \{R(f): f \in W, f \neq 0\}$ ) and if $E_{k+1}$ denotes the set of all $(k+1)$-dimensional vector subspaces of $\mathcal{F}(V)$, then we have

$$
\lambda_{k}(X)=\inf \left\{\Lambda(W): W \in E_{k+1}\right\}
$$

Moreover, let us recall that a rough isometry is an application between metric spaces $\Phi:\left(X_{1}, d_{1}\right) \rightarrow\left(X_{2}, d_{2}\right)$ such that there exist some constants $a \geqslant 1, b \geqslant 0$, $\tau \geqslant 0$ satisfying

$$
\forall x_{1}, y_{1} \in X_{1}, \quad a^{-1} d_{1}\left(x_{1}, y_{1}\right)-b \leqslant d_{2}\left(\Phi\left(x_{1}\right), \Phi\left(y_{1}\right)\right) \leqslant a d_{1}\left(x_{1}, y_{1}\right)+b
$$

and $\cup_{x \in X_{1}} B(\Phi(x), \tau)=X_{2}$ (see [8], p. 142). The constants $a, b$ and $\tau$ will be referred as the constants of rough isometry.

THEOREM 2.1. Let $X$ and $Y$ be finite, connected graphs. Then, for each rough isometry between $X$ and $Y$, there exist positive constants $c$ and $C$ depending only on $v_{X}, v_{Y}$ and on the constants of rough isometry such that

$$
c \leqslant \frac{\lambda_{k}(X)}{\lambda_{k}(Y)} \leqslant C
$$

for all $k<\min \{|X|,|Y|\}$.

Note that the constants $c$ and $C$ are independent of $k,|X|$ and $|Y|$.

Proof. It suffices to prove that it exists $C>0$ such that

$$
\lambda_{k}(X) \leqslant C \lambda_{k}(Y)
$$

We proceed in two steps. 
First, we show that it exists a constant $c^{\prime}>0$ independent of $k$, such that if $\lambda_{k}(Y) \leqslant c^{\prime}$ then (1) is true for some $C>0$. Let $X$ and $Y$ denote also the set of vertices of the respective graphs. Let $\Phi: X \rightarrow Y$ be a rough isometry. To each $f: Y \rightarrow \mathbb{R}$, we associate

$$
\Phi^{*} f=f \circ \Phi: X \rightarrow \mathbb{R}
$$

It can be shown that, there are positive constants $c_{1}, c_{2}, c_{3}, c_{4}$ depending only on $v_{X}, v_{Y}$ and on the constants of rough isometry such that

$$
\begin{aligned}
& \left\|\Phi^{*} f\right\|^{2} \leqslant c_{1}\|f\|^{2}, \\
& \left\|d\left(\Phi^{*} f\right)\right\|^{2} \leqslant c_{2}\|d f\|^{2}, \\
& \|f\|^{2} \leqslant c_{3}\|d f\|^{2}+c_{4}\left\|\Phi^{*} f\right\|^{2}
\end{aligned}
$$

(see [8], lemma VI.5.2 and VI.5.4).

Then, consider $f_{0}, \ldots, f_{k}: Y \rightarrow \mathbb{R}$ eigenfunctions associated to $\lambda_{0}(Y), \ldots$, $\lambda_{k}(Y)$ and the corresponding functions on $X, \Phi^{*} f_{0}, \ldots, \Phi^{*} f_{k}: X \rightarrow \mathbb{R}$. Denote by $V$ the subspace spanned by the $f_{i}$ 's, $V=\left\langle f_{0}, \ldots, f_{k}\right\rangle$ and by $W$ the corresponding space, $W=\left\langle\Phi^{*} f_{0}, \ldots, \Phi^{*} f_{k}\right\rangle$.

If $\lambda_{k}(Y) \leqslant c^{\prime}=\left(2 c_{3}\right)^{-1}$ then $W$ is of dimension $k+1$ : indeed, let $g=$ $\sum_{i=0}^{k} a_{i} \Phi^{*} f_{i}$ with at least one nonzero $a_{i}$. In fact, $g$ can be rewritten as $g=\Phi^{*} f$ with $f=\sum_{i=0}^{k} a_{i} f_{i}$. So, $f$ is a nonzero function of $V$ and satisfies $\|\mathrm{d} f\|^{2} \leqslant$ $\lambda_{k}(Y)\|f\|^{2}$. Then by (4)

$$
\|g\|^{2}=\left\|\Phi^{*} f\right\|^{2} \geqslant c_{4}^{-1}\left(\|f\|^{2}-c_{3}\|d f\|^{2}\right) \geqslant c_{4}^{-1}\|f\|^{2}\left(1-c_{3} \lambda_{k}(Y)\right)
$$

In particular, as $\lambda_{k}(Y) \leqslant c^{\prime}=\left(2 c_{3}\right)^{-1}$, the function $g$ is not zero and this implies that $W$ is $(k+1)$-dimensional. Moreover, under the same assumption on $\lambda_{k}(Y)$ and using (3) and (5), we have $R\left(\Phi^{*} f\right) \leqslant 2 c_{2} c_{4} R(f)$. Finally, we get

$$
\begin{aligned}
\lambda_{k}(X) & \leqslant \sup \{R(g) \mid g \in W-\{0\}\} \\
& =\sup \left\{R\left(\Phi^{*} f\right) \mid f \in V-\{0\}\right\} \\
& \leqslant 2 c_{2} c_{4} \lambda_{k}(Y)
\end{aligned}
$$

It remains to show that (1) is still true if $\lambda_{k}(Y)>c^{\prime}=\left(2 c_{3}\right)^{-1}$. But by this assumption, we have $\lambda_{k}(X) \leqslant \lambda_{k}(X) \lambda_{k}(Y) c^{\prime-1}$. So, in order to conclude, we need an upper bound on $\lambda_{k}(X)$. For each $f: X \rightarrow \mathbb{R}$, we have

$$
\begin{aligned}
\|\mathrm{d} f\|^{2} & =\sum_{x \in X} \sum_{y \in N(x)}|f(x)-f(y)|^{2} \\
& \leqslant 2 \sum_{x \in X} \sum_{y \in N(x)}\left(|f(x)|^{2}+|f(y)|^{2}\right) \\
& \leqslant 4 v_{X}\|f\|^{2}
\end{aligned}
$$

This implies that $R(f) \leqslant 4 v_{X}$ for all $f \neq 0$ and $\lambda_{k}(X) \leqslant 4 v_{X}$. Finally, we get $\lambda_{k}(X) \leqslant 4 v_{X} c^{\prime-1} \lambda_{k}(Y)$. 


\section{Comparison of Spectra Between a Manifold and Its Discretization}

Let $\left(M^{n}, g\right)$ be a connected, compact Riemannian manifold. Consider the Laplacian associated to $M$

$$
\Delta f=-\operatorname{div}(\operatorname{grad} f)
$$

and denote its spectrum by

$$
\operatorname{Spec}(M)=\left\{0=\lambda_{0}(M)<\lambda_{1}(M) \leqslant \ldots\right\}
$$

The characterization of the eigenvalues we will use subsequently is given by Rayleigh quotients and Min-Max Theorem (see [16], p. 269, Min-Max Theorem). So for $F: M \rightarrow \mathbb{R}$ define the Rayleigh quotient of $F$ to be

$$
R(F)=\frac{\|\mathrm{d} F\|^{2}}{\|F\|^{2}}=\frac{\int_{M}\|\mathrm{~d} F(x)\|^{2} \mathrm{~d} V(x)}{\int_{M} F(x)^{2} \mathrm{~d} V(x)}
$$

where $d V$ denotes the volume form on $(M, g)$. Then, for any $(k+1)$-dimensional vector subspace $W^{(k+1)}$ of the vector space $\mathcal{C}^{\infty}(M)$ of smooth functions on $M$, the $k$ th eigenvalue of $M$ satisfies

$$
\lambda_{k}(M) \leqslant \sup \left\{R(F): F \in W^{(k+1)}, F \neq 0\right\}
$$

So, if $\Lambda(W)$ denotes the supremum of Rayleigh quotients of nonzero functions in $W$ (i.e. $\Lambda(W)=\sup \{R(F): F \in W, F \neq 0\}$ ) and if $E_{k+1}$ denotes the set of all $(k+1)$-dimensional vector subspaces of $\mathcal{C}^{\infty}(M)$, then we have

$$
\lambda_{k}(M)=\inf \left\{\Lambda(W): W \in E_{k+1}\right\}
$$

Now, we associate a graph to a Riemannian manifold following [8] (Section V.3.2). Let $\left(M^{n}, g\right)$ be a connected compact $n$-dimensional Riemannian manifold. A discretization of $M$, of mesh $\varepsilon>0$, is a graph $X=(V, E)$ such that the set $V$ of vertices is a maximal $\varepsilon$-separated subset of $M$ (so it verifies that for any $v$, $w \in V, v \neq w$, we have $d(v, w) \geqslant \varepsilon$ and $\left.\cup_{v \in V} B(v, \varepsilon)=M\right)$. Moreover, the graph structure of $X$ is entirely determined by the collection of neighbours that we define as follows. For each $v \in V, w \in V$ is a neighbour of $v$, if $0<d(v, w)<3 \varepsilon$ (see [8], p. 140). We denote by $N(v)$ the set of neighbours of $v$.

Furthermore, $X$ is roughly isometric to $M$ (see [8], p. 147). So we will use on $X$ the metric induced by $M$ rather than the path metric.

Fix once and for all $\varepsilon$ smaller than $\frac{1}{2} \operatorname{Inj}(M)$. Denote by $\kappa \geqslant 0$ a constant such that $\operatorname{Ricci}(M, g) \geqslant-(n-1) \kappa g$. Then, by the Bishop-Gromov comparison theorem, $v_{X}$ is bounded above by a constant depending only on $n, \kappa$ and $\varepsilon$. So we can assume $v_{X}=v(n, \kappa, \varepsilon)$. Furthermore, using Croke's Inequality (cf. [8], p. 136) and Bishop's comparison theorem, we have

$$
\frac{1}{V_{-\kappa}(\varepsilon)} \operatorname{Vol}(M) \leqslant|V| \leqslant \frac{2^{n}}{\varepsilon^{n} c(n)} \operatorname{Vol}(M)
$$


where $V_{-\kappa}(\varepsilon)$ denotes the volume of the ball of radius $\varepsilon$ in the simply connected space of constant sectional curvature $-\kappa$ and of dimension $n$.

The goal of this section is to $\operatorname{compare} \operatorname{Spec}(X)$ and $\operatorname{Spec}(M)$ using the same idea as in the case of roughly isometric graphs. So we have to associate functions on $M$ to functions on $V$ and vice versa. This leads us to use the smoothing and the discretization applications considered in Chavel's book. Let us recall the main definitions and results from [8] (Section VI.5) we will need.

First, to go from the discretization $X=(V, E)$ to the manifold $M$, we need to smooth discrete functions.

DEFINITION 3.1. Let $\left\{\phi_{v}\right\}_{v \in V}$ be a partition of unity subordinate to the cover $\{B(v, 2 \varepsilon)\}_{v \in V}$ of $M$. Then for each $f: V \rightarrow \mathbb{R}$, the smoothing of $f$ is defined by $F=\mathcal{S} f: M \rightarrow \mathbb{R}$

$$
(\mathcal{S} f)(x)=\sum_{v \in V} \phi_{v}(x) f(v)
$$

LEMMA 3.2. There exist positive constants $c_{1}$ and $c_{2}$ depending only on $n, \kappa$ and on the mesh of the discretization such that

$$
\begin{aligned}
& \|\mathcal{S} f\|^{2} \leqslant c_{1}\|f\|^{2}, \\
& \|\mathrm{~d}(\mathcal{S} f)\|^{2} \leqslant c_{2}\|\mathrm{~d} f\|^{2} .
\end{aligned}
$$

Proof. See [8], Section VI.5.2.

Then, to go in the other direction, we want to discretize smooth functions.

DEFINITION 3.3. For each $F: M \rightarrow \mathbb{R}$ the discretization of $F$ is defined by $f=\mathcal{D} F: V \rightarrow \mathbb{R}$

$$
\mathcal{D} F(v)=\frac{1}{\operatorname{Vol}(B(v, 3 \varepsilon))} \int_{B(v, 3 \varepsilon)} F(x) \mathrm{d} V(x)
$$

where $\mathrm{d} V$ denotes the volume form on $(M, g)$.

LEMMA 3.4. There exist positive constants $C_{1}$ and $C_{2}$ depending only on $n, \kappa$ and on the mesh of the discretization such that

$$
\begin{aligned}
& \|\mathcal{D} F\|^{2} \leqslant C_{1}\|F\|^{2} \\
& \|\mathrm{~d}(\mathcal{D} F)\|^{2} \leqslant C_{2}\|\mathrm{~d} F\|^{2}
\end{aligned}
$$

Proof. See [8], Section VI.5.1.

Finally, compose $\mathcal{S}$ and $\mathcal{D}$ and look at how it differs from the identity. 
LEMMA 3.5. There exist positive constants $c_{3}$ and $C_{3}$ depending only on $n, \kappa$ and on the mesh of the discretization such that

$$
\begin{aligned}
& \|f-\mathcal{D} \mathcal{S} f\|^{2} \leqslant c_{3}\|\mathrm{~d} f\|^{2} \\
& \|F-\mathcal{S D} F\|^{2} \leqslant C_{3}\|\mathrm{~d} F\|^{2}
\end{aligned}
$$

Proof. see [8], Sections VI.5.4 and VI.5.5.

We now state and prove the main technical theorem of this note.

DEFINITION 3.6. For $\kappa \geqslant 0$ and $r_{0}>0$ define $\mathcal{M}\left(n, \kappa, r_{0}\right)$ as the set of all connected, compact $n$-dimensional Riemannian manifolds $\left(M^{n}, g\right)$ with Ricci curvature and injectivity radius uniformly bounded below, i.e. with $\operatorname{Ricci}(M, g) \geqslant$ $-(n-1) \kappa g$ and $\operatorname{Inj}(M) \geqslant r_{0}$.

THEOREM 3.7. There exist positive constants $c, C$ such that for all $M \in$ $\mathcal{M}\left(n, \kappa, r_{0}\right)$ and for any discretization $X$ of $M$ (with mesh $\left.<\frac{1}{2} r_{0}\right)$, we have

$$
c \leqslant \frac{\lambda_{k}(M)}{\lambda_{k}(X)} \leqslant C
$$

for all $k<|X|$.

The constants $c$ and $C$ depend only on $n, \kappa$ and on the mesh of $X$.

In particular, if we fix the mesh equal to $\frac{1}{4} r_{0}$, then these constants depend only on the local geometry of $M$, i.e. on $n, \kappa$ and $r_{0}$. Note moreover that all constants are independent of $k$.

Theorem 3.7 is a direct consequence of Theorem 3.8 and Theorem 3.9. In fact, the proof of Theorem 3.7 goes in two steps as for the discrete case. The first step deals in some sense with small eigenvalues; we show that Theorem 3.7 is true for eigenvalues smaller than some constant, using Chavel's results (see Theorem 3.8). The second step of the proof of Theorem 3.7 consists in showing that Theorem 3.7 is true even if the eigenvalues are 'big', that is to say bigger than the constants appearing in Theorem 3.8. The proof here is really different from the previous one and uses basic facts on eigenvalues of Laplacian (see Theorem 3.9).

THEOREM 3.8. There exist positive constants $a, A, c^{\prime}$ and $C^{\prime}$ such that for all $M \in \mathcal{M}\left(n, \kappa, r_{0}\right)$ and for any discretization $X$ of $M$ (with mesh $\left.<\frac{1}{2} r_{0}\right)$, we have

(i) If $\lambda_{k}(X) \leqslant a$, then $\frac{\lambda_{k}(M)}{\lambda_{k}(X)} \leqslant c^{\prime}$,

(ii) If $\lambda_{k}(M) \leqslant A$, then $\frac{\lambda_{k}(X)}{\lambda_{k}(M)} \leqslant C^{\prime}$

for all $k<|X|$.

The constants $a, A, c^{\prime}$ and $C^{\prime}$ depend only on $n, \kappa$ and on the mesh of $X$. 
Proof. (i) The idea of the proof is exactly the same as in Theorem 2.1, using the above lemma in order to bound Rayleigh quotients. Let $M \in \mathcal{M}\left(n, \kappa, r_{0}\right)$ and $X=(V, E)$ a discretization with mesh $<\frac{1}{2} r_{0}$. Then, consider $f_{0}, \ldots, f_{k}: V \rightarrow \mathbb{R}$ eigenfunctions associated to the first $k+1$ eigenvalues of $X$. Denote by $W$ the subspace spanned by these eigenfunctions. Smooth each $f_{i}$ to obtain $F_{0}=\mathcal{S} f_{0}$, $\ldots, F_{k}=\mathcal{S} f_{k}: M \rightarrow \mathbb{R}$ and $\mathcal{S} W$ the subspace spanned by the $F_{i}$ 's.

Then, if $\lambda_{k}(X)<a=\frac{1}{4} c_{3}^{-1}, \mathcal{S} W$ is $(k+1)$-dimensional. In order to prove this fact, let $F$ be an element of $\mathcal{S} W, F=\sum_{i=0}^{k} a_{i} F_{i}$ with at least one non-zero coefficient. In fact, $F$ is the smoothing of a discrete $f \in W$ such that $f=\sum_{i=0}^{k} a_{i} f_{i}$ and by (10), we have $\|f-\mathcal{D S} f\|^{2} \leqslant c_{3}\|d f\|^{2}$. So the norm of $F$ satisfies (by (8))

$$
\|F\| \geqslant C_{1}^{-\frac{1}{2}}\|\mathcal{D} F\| \geqslant C_{1}^{-\frac{1}{2}}(\|f\|-\|f-\mathcal{D} \mathcal{S} f\|) \geqslant C_{1}^{-\frac{1}{2}}\left(\|f\|-c_{3}^{\frac{1}{2}}\|d f\|\right)
$$

But $f$ is a nonzero function belonging to the subspace $W$, so it satisfies $\|d f\| \leqslant$ $\sqrt{\lambda_{k}(X)}\|f\|$ and by assumption on $\lambda_{k}(X)$ and by (12), we get

$$
\|F\| \geqslant \frac{1}{2} C_{1}^{-\frac{1}{2}}\|f\|
$$

As the eigenfunctions of $X$ are linearly independent, this shows that the dimension of $\mathcal{S} W$ is the same as the dimension of $W$, that is to say $k+1$. Moreover, under the same assumption on $\lambda_{k}(X)$ and using (13), we obtain

$$
R(\mathcal{S} f) \leqslant c^{\prime} R(f)
$$

for all $f \in W-\{0\}$. This leads now to the conclusion. Using the Min-Max Theorem, we have

$$
\begin{aligned}
\lambda_{k}(M) & \leqslant \sup \{R(F) \mid F \in \mathcal{S} W-\{0\}\} \\
& \leqslant c^{\prime} \sup \{R(f) \mid f \in W-\{0\}\} \\
& =c^{\prime} \lambda_{k}(X)
\end{aligned}
$$

and this ends the proof of the theorem. The second part of the proof can be carried out exactly in the same way, because of the symmetry of the results concerning the smoothing and the discretization of functions. So it would not bring more informations to do it here.

THEOREM 3.9. Let $a$ and $A$ as in Theorem 3.8. Then, there exist positive constants $c^{\prime \prime}$ and $C^{\prime \prime}$ such that for all $M \in \mathcal{M}\left(n, \kappa, r_{0}\right)$ and for any discretization $X$ of $M$ (with mesh $\left.<\frac{1}{2} r_{0}\right)$, we have

(i) If $\lambda_{k}(X)>a$, then $\frac{\lambda_{k}(M)}{\lambda_{k}(X)} \leqslant c^{\prime \prime}$.

(ii) If $\lambda_{k}(M)>A$, then $\frac{\lambda_{k}(X)}{\lambda_{k}(M)} \leqslant C^{\prime \prime}$,

for all $k<|X|$.

The constants $c^{\prime \prime}$ and $C^{\prime \prime}$ depend only on $n, \kappa$ and on the mesh of $X$. 
Proof. (i) As $\lambda_{k}(X)>a$, then $\lambda_{k}(M)<a^{-1} \lambda_{k}(X) \lambda_{k}(M)$. So, it suffices to show that $\lambda_{k}(M) \leqslant \lambda_{1}^{\kappa}\left(\frac{\varepsilon}{2}\right)$, where $\lambda_{1}^{\kappa}\left(\frac{\varepsilon}{2}\right)$ denotes the first nonzero eigenvalue of the Dirichlet problem on the ball of radius $\frac{\varepsilon}{2}$ in the simply connected space of constant sectional curvature $-\kappa$ and of same dimension as $M$. We prove this result as follows. For each vertex $v_{i}$ of $X, i \geqslant 1$, we can consider $f_{i}: M \rightarrow \mathbb{R}$ the first eigenfunction of the Dirichlet problem for the ball $\bar{B}\left(v_{i}, \frac{\varepsilon}{2}\right)$ extended by 0 outside the ball. By Cheng's comparison Theorem $R\left(f_{i}\right) \leqslant \lambda_{1}^{\kappa}\left(\frac{\varepsilon}{2}\right)$ (see [9], p. 74).

Now, consider $V_{i}$ the subspace spanned by $f_{1}, \ldots, f_{i}$. As the balls of radius $\varepsilon / 2$ are disjoint, the $f_{i}$ 's are orthogonal and so $V_{i}$ is of dimension $i$. We can apply Min-Max Theorem and get for all $k<|X|$

$$
\begin{aligned}
\lambda_{k}(M) & \leqslant \sup \left\{R(f): f \in V_{k+1}, f \neq 0\right\} \\
& =\sup \left\{\frac{\sum a_{i}^{2}\left\|d f_{i}\right\|^{2}}{\sum a_{i}^{2}\left\|f_{i}\right\|^{2}}: f=\sum a_{i} f_{i} \in V_{k+1}, f \neq 0\right\} \\
& \leqslant \sup \left\{\frac{\sum a_{i}^{2} \lambda_{1}^{\kappa}\left(\frac{\varepsilon}{2}\right)\left\|f_{i}\right\|^{2}}{\sum a_{i}^{2}\left\|f_{i}\right\|^{2}}: f=\sum a_{i} f_{i} \in V_{k+1}, f \neq 0\right\} \\
& \leqslant \lambda_{1}^{\kappa}\left(\frac{\varepsilon}{2}\right)
\end{aligned}
$$

Finally, we get $\lambda_{k}(M)<a^{-1} \lambda_{1}^{\kappa}\left(\frac{\varepsilon}{2}\right) \lambda_{k}(X)$.

(ii) As $\lambda_{k}(M)>A$, then $\lambda_{k}(X) \leqslant A^{-1} \lambda_{k}(M) \lambda_{k}(X)$. We have seen in Theorem 2. that $\lambda_{k}(X) \leqslant 4 v_{X}$. So we get $\lambda_{k}(X) \leqslant 4 A^{-1} v_{X} \lambda_{k}(M)$.

\section{Application to the Spectrum of a Tower of Coverings}

As a first application, we will discuss the following theorem.

THEOREM 4.1 Let $\left(M^{n}, g\right)$ be a compact $n$-dimensional Riemannian manifold. Let $\left\{M_{i}\right\}_{i} \geqslant 1$ be a family of finite-sheeted covering spaces of $M$ with induced Riemannian metric. Let $\Gamma_{i}$ be the Schreier graph of the subgroup $\pi_{1}\left(M_{i}\right)$ of $\pi_{1}(M)$. Then, there exist constants $c, C>0$ such that for all $k<\left|\Gamma_{i}\right|$

$$
c \leqslant \frac{\lambda_{k}\left(M_{i}\right)}{\lambda_{k}\left(\Gamma_{i}\right)} \leqslant C
$$

In particular, for all $k$

$$
\lambda_{k}\left(M_{i}\right) \rightarrow 0 \quad \text { when } i \rightarrow \infty \Longleftrightarrow \lambda_{k}\left(\Gamma_{i}\right) \rightarrow 0 \text { when } i \rightarrow \infty .
$$

For $k=1$, it is exactly the result of Brooks (see [2], Theorem 1).

We prove the result in two steps. First, we associate to $M_{i}$ a discretization $X_{i}$ of sufficiently small mesh, in order to compare $\operatorname{Spec}\left(M_{i}\right)$ to $\operatorname{Spec}\left(X_{i}\right)$ (as in Section 3). Secondly, we show that $X_{i}$ and $\Gamma_{i}$ are roughly isometric, which allows us to apply 
Theorem 2.1 to $\operatorname{Spec}\left(\Gamma_{i}\right)$ and $\operatorname{Spec}\left(X_{i}\right)$, so that we obtain the desired result between $\operatorname{Spec}\left(M_{i}\right)$ and $\operatorname{Spec}\left(\Gamma_{i}\right)$.

The first step is really a direct application of Theorem 3.7 and is stated in Theorem 4.2.

THEOREM 4.2 Let $M$ and $\left\{M_{i}\right\}_{i} \geqslant 1$ be as in Theorem 4.1. Let $X$ be adiscretization of $M$ (with mesh $<\frac{1}{2} \operatorname{Inj}(M)$ ) and lift it to $M_{i}$ to obtain a discretization $X_{i}$ of $M_{i}$. Then, there exist positive constants $c$ and $C$ independent of $i$ such that for all $k<\left|X_{i}\right|$.

$$
c \leqslant \frac{\lambda_{k}\left(M_{i}\right)}{\lambda_{k}\left(X_{i}\right)} \leqslant C
$$

In particular, for all $k$

$$
\lambda_{k}\left(M_{i}\right) \rightarrow 0 \quad \text { when } i \rightarrow \infty \Longleftrightarrow \lambda_{k}\left(X_{i}\right) \rightarrow 0 \text { when } i \rightarrow \infty
$$

Proof. If $\left(M^{n}, g\right) \in \mathcal{M}\left(n, \kappa, r_{0}\right)$, then $M_{i} \in \mathcal{M}\left(n, \kappa, r_{0}\right)$, as $M_{i}$ is provided with the induced Riemannian metric. Moreover, $X_{i}$ is a discretization of $M_{i}$ with same mesh of $X$ smaller than $\frac{1}{2} r_{0}$. So we can apply Theorem 3.7 to each pair $\left(M_{i}, X_{i}\right)$ and get constants independent of $i$.

In the second step, we have to compare the Schreier graph $\Gamma_{i}$ appearing in Theorem 4.1 to the discretization $X_{i}$ of $M_{i}$ appearing in Theorem 4.2. This is the next result.

THEOREM 4.3 Let $\left\{\left(X_{i}, \Gamma_{i}\right)\right\}_{i} \geqslant 1$ be as in Theorems 4.1 and 4.2. Then, there exist positive constants $c$ and $C$ independent of $i$ such that for all $k<\left|\Gamma_{i}\right|$

$$
c \leqslant \frac{\lambda_{k}\left(X_{i}\right)}{\lambda_{k}\left(\Gamma_{i}\right)} \leqslant C
$$

In particular, for all $k$

$$
\lambda_{k}\left(X_{i}\right) \rightarrow 0 \text { when } i \rightarrow \infty \Longleftrightarrow \lambda_{k}\left(\Gamma_{i}\right) \rightarrow 0 \text { when } i \rightarrow \infty
$$

Proof. Geometrically, the graph $\Gamma_{i}$ corresponds to the lift of a graph $\Gamma$ in $M$, where $\Gamma$ consists of a unique point (see [1], p. 254, for a definition of Schreier graphs). As $M$ is compact, $\Gamma$ and $X$ are roughly isometric (see [8], p. 147). Let us call $\Gamma, \Gamma_{i}$ and $X, X_{i}$ the set of vertices of the respective graphs too and $\Phi: \Gamma \rightarrow X$ a rough isometry such that $d(g, \Phi(g))<\varepsilon$. We can lift $\Phi$ and get $\Phi_{i}: \Gamma_{i} \rightarrow X_{i}$ in the following way. If $g \in \Gamma_{i}$, then by construction of $X_{i}$, there exists $\Phi_{i}(g)=x$ with $\pi_{i}(x)=\Phi\left(\pi_{i}(g)\right)$ and $d(x, g)<\varepsilon$ (where $\pi_{i}$ denotes the canonical projection of $M_{i}$ onto $M$ ). Clearly, $\Phi_{i}$ is a rough isometry with same constants of rough isometry as $\Phi$. So we can apply Theorem 2.1 to each pair $\left(X_{i}, \Gamma_{i}\right)$ and get constants independent of $i$. 
Note that, as any two discretizations of a compact manifold are roughly isometric, then we can replace $\Gamma_{i}$ by the lift of any discretization of $M$ and Theorem 4.1 is still true.

\section{Gromov-Hausdorff Close Manifolds have Comparable Spectra}

Another application of Theorem 3.7 is the following result.

THEOREM 5.1. Let $\left(M^{m}, g_{M}\right) \in \mathcal{M}\left(m, \kappa, r_{0}\right)$ and $\left(N^{n}, g_{N}\right) \in \mathcal{M}\left(n, \kappa, r_{0}\right)$. Suppose that the Gromov-Hausdorff distance between $M$ and $N$ is smaller than $\eta>0$. Then, there exist positive constants $c$ and $C$ (depending only on $\eta, \kappa, r_{0}$ and on the dimensions) and $K>0$ (proportional to the volume of $M$ and $N$ ) such that for all $k<K$

$$
c \leqslant \frac{\lambda_{k}(M)}{\lambda_{k}(N)} \leqslant C
$$

and there exist $c^{\prime}$ and $C^{\prime}$ (depending on the dimensions, $\eta, \kappa$ and $r_{0}$ ) such that for all $k \geqslant K$

$$
c^{\prime} \leqslant \frac{\lambda_{k}(M)^{m}}{\lambda_{k}(N)^{n}} \leqslant C^{\prime}
$$

COROLLARY 5.2. Let $M$ and $N$ be two same dimensional compact Riemannian manifolds, i.e. $M, N \in \mathcal{M}\left(n, \kappa, r_{0}\right)$. Suppose that the Gromov-Hausdorff distance between $M$ and $N$ is smaller than $\eta>0$. Then, there exist positive constants $c$ and $C$ (depending only on $\eta, n, \kappa$ and $r_{0}$ ) such that for all $k$

$$
c \leqslant \frac{\lambda_{k}(M)}{\lambda_{k}(N)} \leqslant C
$$

Proof of Theorem 5.1. Recall (from Section 3) that if $X_{M}$ is a discretization of $M \in \mathcal{M}\left(m, \kappa, r_{0}\right)$ of mesh $\varepsilon<\frac{1}{2} r_{0}$, then

$$
\frac{1}{V_{-\kappa}(\varepsilon)} \operatorname{Vol}(M) \leqslant\left|X_{M}\right| \leqslant \frac{2^{m}}{\varepsilon^{m} c(m)} \operatorname{Vol}(M)
$$

Let $X_{M}$ be a discretization of $M \in \mathcal{M}\left(m, \kappa, r_{0}\right)$ and $X_{N}$ a discretization of $N \in \mathcal{M}\left(n, \kappa, r_{0}\right)$ with same mesh $\varepsilon<\frac{1}{2} r_{0}$. Moreover, choose $\varepsilon<\frac{1}{2} r_{0}$ such that

$$
\min \left\{\left|X_{M}\right|,\left|X_{N}\right|\right\} \geqslant \max \left\{\operatorname{Vol}(M) \frac{2^{m+2}}{c(m) r_{0}^{m}}, \operatorname{Vol}(N) \frac{2^{n+2}}{c(n) r_{0}^{n}}\right\}
$$

( $\varepsilon$ depend only on $m, n, \kappa$ and $r_{0}$ ).

Then, the proof is done in two steps. First, if $k<\min \left\{\left|X_{M}\right|,\left|X_{N}\right|\right\}$, we will apply Theorem 2.1 and Theorem 3.7 (as will follow) and second, for $k \geqslant$ $\min \left\{\left|X_{M}\right|,\left|X_{N}\right|\right\}$, we will use a result of Buser (Theorem 6.2 in [6]). 
Consider the case $k<\min \left\{\left|X_{M}\right|,\left|X_{N}\right|\right\}$. By definition of the GromovHausdorff distance (see [12]) there exist $Z$ a Riemannian manifold and two isometric embeddings $f: M \rightarrow Z, g: N \rightarrow Z$ such that $\cup_{x \in M} B(f(x), \eta) \supset g(N)$ and $\cup_{y \in N} B(g(y), \eta) \supset f(M)$. Then, $X_{M}$ and $X_{N}$ are roughly isometric via $\Phi: X_{M} \rightarrow X_{N}$ defined as follows. For each $x \in X_{M}$ there exist $x^{\prime} \in N$ and $x^{\prime \prime} \in X_{N}$ such that $d\left(f(x), g\left(x^{\prime}\right)\right)<\eta$ and $d\left(x^{\prime}, x^{\prime \prime}\right)<\varepsilon$. Then define $\Phi(x)=x^{\prime \prime}$ so that $d(f(x), g(\phi(x)))<\eta+\varepsilon$. Then, we clearly have that

$$
d(x, y)-2(\eta+\varepsilon)<d(\Phi(x), \Phi(y))<d(x, y)+2(\eta+\varepsilon)
$$

Moreover, if $z \in X_{N}$ then there exist $y \in M$ and $x \in X_{M}$ such that $d(g(z), f(y))<\eta$ and $d(x, y)<\varepsilon$. This implies that

$$
d(\Phi(x), z) \leqslant d(g(\Phi(x)), f(x))+d(f(x), f(y))+d(f(y), g(z))<2(\eta+\varepsilon)
$$

and so $\cup_{x \in X_{M}} B(\Phi(x), 2(\eta+\varepsilon)) \supset X_{N}$. Note that the constants of rough isometry depend only on $\varepsilon$ and $\eta$. To conclude this first part, it suffices to apply Theorem 3.7 to $\left(M, X_{M}\right)$ and $\left(N, X_{N}\right)$ and Theorem 2.1 to $\left(X_{M}, X_{N}\right)$.

Now, consider the case $k \geqslant \min \left\{\left|X_{M}\right|,\left|X_{N}\right|\right\}$. In this case and by assumption on $\varepsilon$, we can apply Theorem 6.2 of [6] to $M$ and $N$ which leads to the result

$$
\left(\frac{k}{\operatorname{Vol}(M)}\right)^{\frac{2}{m}} c_{1} \leqslant \lambda_{k}(M) \leqslant\left(\frac{k}{\operatorname{Vol}(M)}\right)^{\frac{2}{m}} c_{2}
$$

where $c_{1}$ and $c_{2}$ are constants depending on $m, \kappa$ and $r_{0}$. Similarly,

$$
\left(\frac{k}{\operatorname{Vol}(N)}\right)^{\frac{2}{n}} c_{3} \leqslant \lambda_{k}(N) \leqslant\left(\frac{k}{\operatorname{Vol}(N)}\right)^{\frac{2}{n}} c_{4},
$$

where $c_{3}$ and $c_{4}$ are constants depending on $n, \kappa$ and $r_{0}$. Putting both inequalities together, we get that there exist constants $c_{5}$ and $c_{6}$ depending only on the dimensions, $\kappa$ and $r_{0}$ such that

$$
c_{5}\left(\frac{\operatorname{Vol}(M)}{\operatorname{Vol}(N)}\right)^{2} \leqslant \frac{\lambda_{k}(M)^{m}}{\lambda_{k}(N)^{n}} \leqslant c_{6}\left(\frac{\operatorname{Vol}(M)}{\operatorname{Vol}(N)}\right)^{2}
$$

Furthermore, we have seen in Section 3 that there exist constants $c_{7}, c_{8}, c_{9}$ and $c_{10}$ depending only on the dimensions, $\kappa$ and $r_{0}$ such that

$$
\begin{aligned}
& c_{7}\left|X_{M}\right| \leqslant \operatorname{Vol}(M) \leqslant c_{8}\left|X_{M}\right|, \\
& c_{9}\left|X_{N}\right| \leqslant \operatorname{Vol}(N) \leqslant c_{10}\left|X_{N}\right| .
\end{aligned}
$$

As we have shown in the first part of the proof the discretizations are roughly isometric (the constants of rough isometry depend only on $\varepsilon$ and $\eta$ ). This implies that there exist constants $c_{11}$ and $c_{12}$ depending on $\varepsilon$ and $\eta$ such that

$$
c_{11} \leqslant \frac{\left|X_{M}\right|}{\left|X_{N}\right|} \leqslant c_{12}
$$


These last inequalities give us upper and lower bounds (depending only on $\eta, m$, $n, \kappa$ and $\left.r_{0}\right)$ for $\operatorname{Vol}(M) / \operatorname{Vol}(N)$.

Finally, we get that there exist constants $c, C>0$ depending only on $\eta, m, n, \kappa$ and $r_{0}$ such that for all $k$

$$
c \leqslant \frac{\lambda_{k}(M)^{m}}{\lambda_{k}(N)^{n}} \leqslant C .
$$

In particular, the constants do not depend on $k$.

\section{An Example}

Let us now discuss an example in order to show that the assumption on the injectivity radius in Theorem 5.1 is necessary. Consider $M_{\varepsilon}$ the manifold obtained by taking two hyperbolic cylinders, gluing them together at both ends and 'smoothing the angles'.

More precisely, for $0<\varepsilon<1$ take

$$
M_{\varepsilon}=\left[-1-\rho_{\varepsilon} ; 1+\rho_{\varepsilon}\right] \times \mathbb{S}^{1} / \sim
$$

where $\sim$ identifies the ends (i.e. $\left(\rho_{1}, t_{1}\right) \sim\left(\rho_{2}, t_{2}\right)$ if and only if $\rho_{1}=\rho_{2}= \pm\left(1+\rho_{\varepsilon}\right)$ and $\left.t_{1}=t_{2}\right)$ and $\rho_{\varepsilon}=\operatorname{arccosh}\left(\varepsilon^{-\frac{1}{2}}\right)$. Provide $M_{\varepsilon}$ with the Riemannian metric

$$
\mathrm{d} s^{2}=\mathrm{d} \rho^{2}+f(\rho)^{2} \mathrm{~d} t^{2}
$$

where

$$
f(\rho)= \begin{cases}\varepsilon \cosh \left(\rho+\rho_{\varepsilon}+1\right) & \text { if } \rho \in\left[-1-\rho_{\varepsilon} ;-1\right], \\ \frac{\sqrt{\varepsilon}}{8}\left((1+\sqrt{1-\varepsilon}) \rho^{4}-2(1+3 \sqrt{1-\varepsilon}) \rho^{2}+9+5 \sqrt{1-\varepsilon}\right) & \text { if } \rho \in[-1 ; 1], \\ \varepsilon \cosh \left(-\rho+\rho_{\varepsilon}+1\right) & \text { if } \rho \in\left[1 ; 1+\rho_{\varepsilon}\right] .\end{cases}
$$

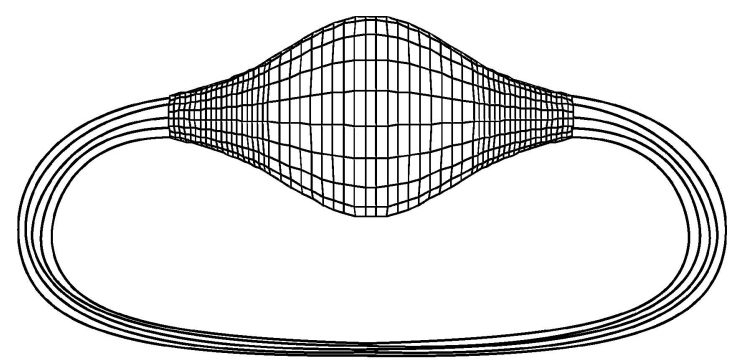

The manifold $M_{\varepsilon}$

Then, $M_{\varepsilon}$ has sectional curvature uniformly bounded (i.e. independently of $\varepsilon$ ) and injectivity radius comparable to $\varepsilon$. Moreover, as $M_{\varepsilon}$ admits an involution and if $D_{\varepsilon}=\left\{(\rho, t): \rho \in\left[-1-\rho_{\varepsilon} ; 0\right], t \in \mathbb{R} / \mathbb{Z}\right\}$, then $\lambda_{1}\left(M_{\varepsilon}\right)$ is either the first nonzero eigenvalue of $D_{\varepsilon}$ for the Neumann problem $\lambda_{1}^{N}\left(D_{\varepsilon}\right)$ or the first eigenvalue of $D_{\varepsilon}$ for the Dirichlet problem $\lambda_{1}^{D}\left(D_{\varepsilon}\right)$, where $D_{\varepsilon}$ is provided with the same 
Riemannian metric as $M_{\varepsilon}$. But $D_{\varepsilon}$ can be provided with an hyperbolic metric $d s^{\prime 2}=d \rho^{2}+\varepsilon^{2} \cosh ^{2}\left(\rho+\rho_{\varepsilon}+1\right) d t^{2}$ and we can easily show that there are positive constants $c_{1}$ and $c_{2}$ independent of $\varepsilon$ such that

$$
c_{1} d s^{2} \leqslant d s^{\prime 2} \leqslant c_{2} d s^{2} .
$$

Then, applying a result of Dodziuk (see [11], Proposition 3.3), we get a constant $c_{3}$ independent of $\varepsilon$ such that

$$
\lambda_{1}^{N, D}\left(D_{\varepsilon}, d s^{2}\right) \geqslant c_{3} \lambda_{1}^{N, D}\left(D_{\varepsilon}, d s^{2}\right)
$$

But, we know that for thin hyperbolic cylinders $\lambda_{1}^{N, D}\left(D_{\varepsilon}, d s^{2}\right) \geqslant \frac{1}{4}$ (see [5], p. 35). Finally, we have shown that $\lambda_{1}\left(M_{\varepsilon}\right) \geqslant c>0$ where $c$ is independent on $\varepsilon$.

Moreover, $M_{\varepsilon}$ is Hausdorff-Gromov close to the circle $S_{\varepsilon}$ of length $2 \rho_{\varepsilon}+2$ and $\lambda_{1}\left(S_{\varepsilon}\right)$ goes to zero when $\varepsilon$ goes to zero too. So

$$
\frac{\lambda_{1}\left(M_{\varepsilon}\right)}{\lambda_{1}\left(S_{\varepsilon}\right)} \rightarrow \infty \quad \text { when } \varepsilon \rightarrow 0
$$

Then, this quotient cannot be bounded and the theorem is not true for the family $\left(M_{\varepsilon}, S_{\varepsilon}\right)$, because the injectivity radius of $M_{\varepsilon}$ is not uniformly bounded below.

\section{References}

1. Bollobás, B.: Modern Graph Theory, Springer, New York, 1998.

2. Brooks, R.: The spectral geometry of a tower of coverings, J. Differential Geom. 23 (1986), 97-107.

3. Brooks, R.: Combinatorial problems in spectral geometry, In: Curvature and Topology of Riemannian Manifolds (Katata 1985), Lecture Notes in Math. 1201, Springer, New York, 1986, pp. 14-32.

4. Burger, M.: Estimation de petites valeurs propres du laplacien d'un revêtement de variétés riemanniennes compactes, C.R. Acad. Sci. Paris Ser. I Math. 302(5) (1986), 191-194.

5. Buser, P.: On Cheeger's Inequality, Proc. Sympos. Pure Math. 36, Amer. Math. Soc., Providence, 1980.

6. Buser, P.: A note on the isoperimetric constant, Ann. Sci. Ecole Norm. Sup. 15 (1982), 213-230.

7. Buser, P.: On the bipartition of graphs, Discrete Appl. Math. 9(1) (1984), 105-109.

8. Chavel, I.: Isoperimetric Inequalities, Cambridge University Press, 2001.

9. Chavel, I.: Eigenvalues in Riemannian Geometry, Academic Press, New York, 1984.

10. Cheeger, J. and Colding, T.: On the structure of spaces with Ricci curvature bounded below III, J. Differential Geom. 54 (2000), 37-74.

11. Dodziuk, J.: Eigenvalues of the Laplacian on forms, Proc. Amer. Math. Soc. 85(3) (1982).

12. Gromov, M., rédigé par Lafontaine J. et Pansu P.: Structures métriques pour les variétés riemanniennes, Cedic, 1981.

13. Kanai, M.: Rough isometries, and combinatorial approximations of geometries of non-compact Riemannian manifolds, J. Math. Soc. Japan 37(3) (1985).

14. Kanai, M.: Analytic inequalities, and rough isometries between non-compact Riemannian manifolds, in: Curvature and Topology of Riemannian Manifolds (Katata, 1985), Lecture Notes in Math. 1201, Springer, New York, 1986, pp. 122-137.

15. Lubotsky, A.: Discrete Groups, Expanding Graphs and Invariant Measures, Birkhäuser, Basel, 1994.

16. Sakai, T.: Riemannian Geometry, Amer. Math. Soc., Providence, 1997. 